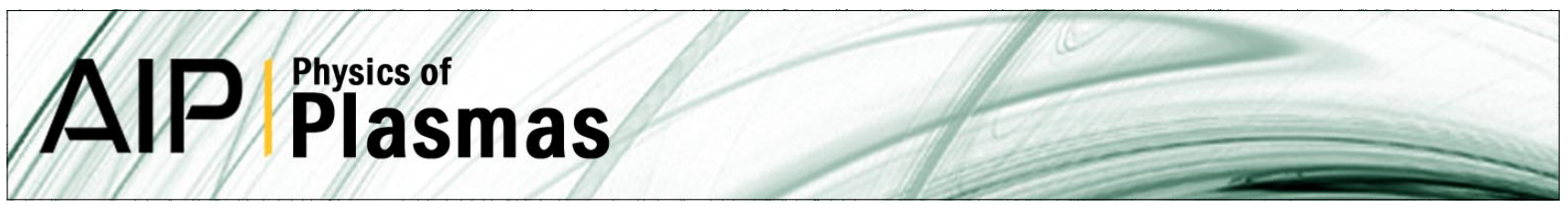

\title{
Properties of convective cells generated in magnetized toroidal plasmas
}

C. Theiler, J. Loizu, I. Furno, A. Fasoli, and P. Ricci

Citation: Phys. Plasmas 19, 082304 (2012); doi: 10.1063/1.4740056

View online: http://dx.doi.org/10.1063/1.4740056

View Table of Contents: http://pop.aip.org/resource/1/PHPAEN/v19/i8

Published by the American Institute of Physics.

\section{Related Articles}

Parallel transport of long mean-free-path plasmas along open magnetic field lines: Plasma profile variation Phys. Plasmas 19, 082310 (2012)

Parallel resistivity and ohmic heating of laboratory dipole plasmas

Phys. Plasmas 19, 082509 (2012)

Influence of charging process and size distribution of dust grain on the electric conductivity of dusty plasma Phys. Plasmas 19, 083703 (2012)

Stationary electron velocity distribution function in crossed electric and magnetic fields with collisions Phys. Plasmas 19, 083503 (2012)

Measurements of parallel electron velocity distributions using whistler wave absorption Rev. Sci. Instrum. 83, 083503 (2012)

\section{Additional information on Phys. Plasmas}

Journal Homepage: http://pop.aip.org/

Journal Information: http://pop.aip.org/about/about_the_journal

Top downloads: http://pop.aip.org/features/most_downloaded

Information for Authors: http://pop.aip.org/authors

\section{ADVERTISEMENT}

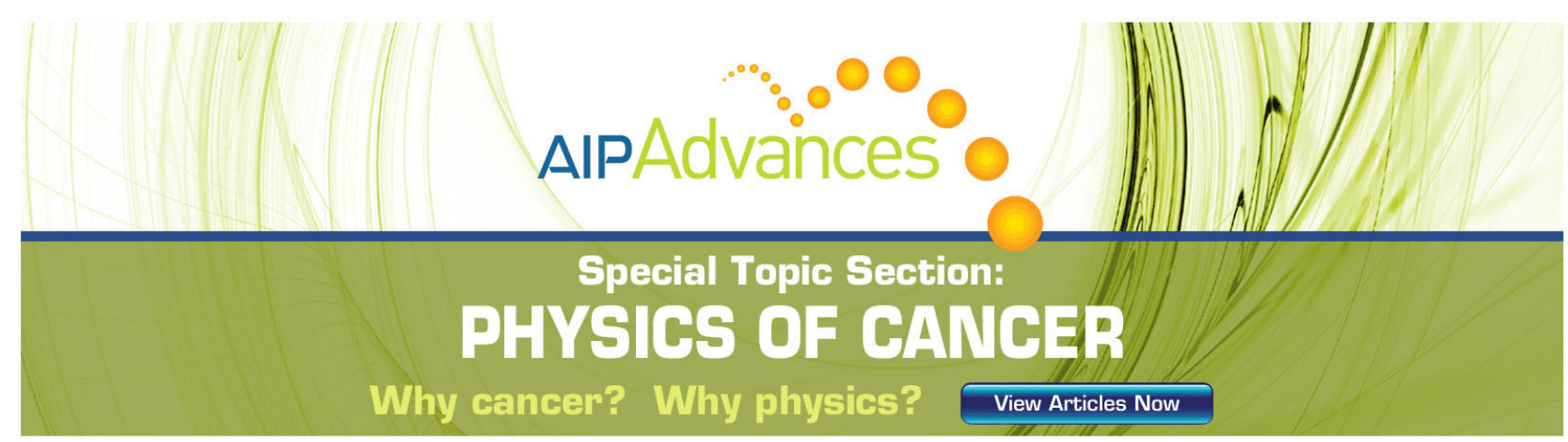




\title{
Properties of convective cells generated in magnetized toroidal plasmas
}

\author{
C. Theiler, ${ }^{\text {a) }}$ J. Loizu, I. Furno, A. Fasoli, and P. Ricci \\ Centre de Recherches en Physique des Plasmas-Ecole Polytechnique Fédérale de Lausanne (EPFL), \\ Association EURATOM-Confédération Suisse, CH-1015 Lausanne, Switzerland
}

(Received 27 May 2012; accepted 13 July 2012; published online 6 August 2012)

\begin{abstract}
Convective cells for turbulence control, generated by means of biased electrodes, are investigated in the simple magnetized toroidal plasmas of TORPEX. A two-dimensional array of 24 electrodes is installed on a metal limiter to test different biasing schemes. This allows influencing significantly both radial and vertical blob velocities. It is shown that these changes agree quantitatively with the flows deduced from the time averaged potential perturbations induced by the biasing. Detailed measurements along and across the magnetic field provide a rather clear picture of the effect of biasing on time averaged profiles. The biased electrodes produce perturbations of the plasma potential and density profiles that are fairly uniform along the magnetic field. Background flows influence the location where potential variations are induced. The magnitude of the achievable potential variations in the plasma is strongly limited by cross-field currents and saturates at large bias voltages once the electrodes draw electron saturation current. A quantitative discussion on the origin of cross-field currents is presented, considering contributions related with diamagnetic drifts, ion inertia, collisions with neutrals, and viscosity. (C) 2012 American Institute of Physics. [http://dx.doi.org/10.1063/1.4740056]
\end{abstract}

\section{INTRODUCTION}

Inside the last closed flux surface of magnetic fusion devices, a low level of cross-field heat transport is desired for good performance. This is not necessarily the case for the open field lines region or scrape-off layer (SOL). Indeed, if the level of cross-field transport in the SOL is low, the heat crossing the last closed flux surface reaches the divertor in a narrow layer along the magnetic field, resulting in a large local heat flux. Handling such steady-state heat loads on the divertor constitutes a serious issue for ITER. ${ }^{1-6}$ It is therefore of interest to develop tools to actively influence the SOL width. It has been proposed that inducing poloidal electric fields and convective cells in the SOL could serve as a method to increase its width and reduce divertor heat loads. ${ }^{7}$ The most direct way to do this is by active toroidal or poloidal asymmetric biasing, using asymmetric divertor biasing schemes or inserting electrodes into the SOL. Parallel currents generated in this way can, if sufficiently large, produce significant magnetic perturbations with additional, potentially beneficial effects. ${ }^{8}$ While active biasing might not be an option in an actual reactor, it allows investigating the physics associated with convective cells. Other methods could eventually be applied to achieve the desired potential variations. Possible candidates are discussed in Refs. 7, 9, and 10.

Toroidal or poloidal asymmetric biasing has already been tested in several tokamaks. In JFT-2M, biasing was applied to 2 out of 14 neighboring inboard divertor plates. ${ }^{11}$ Strong changes in the poloidal electric field could be generated this way in the SOL. Further, significant modifications of the electron heat flux onto the divertor were observed. In MAST, ${ }^{12,13}$ biasing was applied to every other rib of the out-

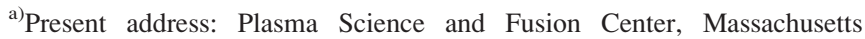
Institute of Technology, Cambridge, MA 02139, USA. Electronic mail: theiler@psfc.mit.edu.
}

board lower divertor in different experimental conditions. This led to a toroidally wavy wetted area on the divertor and modifications of the heat flux width and peak value. In CASTOR, an electrode was immersed in the SOL, leading to the formation of convective cells around the biased flux tube. ${ }^{14}$ In NSTX, an array of four electrodes was installed in the SOL. ${ }^{15}$ Experiments were performed in a wide range of regimes. Strong local effects on the SOL profiles were measured and clear evidence of convective cell formation could be inferred. These experiments have recently been complemented with biased electrodes installed on the divertor of NSTX. ${ }^{16}$

In Ref. 17, we have investigated the basic mechanisms governing the formation of convective cells and their effect on turbulent structures in the magnetized toroidal TORPEX (Refs. 18 and 19) plasmas. With open field lines and curvature driven instabilities, TORPEX includes important aspects of SOL turbulence. At the same time, full access with probes is possible due to relatively low values of density and temperature. Together with a relatively simple geometry and high flexibility, this allows for detailed measurements along and across the magnetic field. Using an array of $3 \times 8$ biasing electrodes, we produced modifications of time-averaged profiles that reveal features of convective cells. Depending on the biasing scheme, we demonstrated that both radial and vertical velocities of turbulent structures (blobs) are significantly modified. We further showed that the effect of biasing on the time-averaged profiles is fairly uniform along the magnetic field, that the magnitude of the induced potential structures is limited by cross-field currents, and its position is shifted with respect to the biased flux tube in the direction of plasma flows. ${ }^{17}$ The aim of this paper is to go beyond the initial proof of principle demonstration and analyze in a quantitative way the details of the mechanisms at play and the limitations that can be associated with them. We show that the changes in blob velocity due to the biasing are in quantitative agreement with modifications of the velocity 
field, calculated from the time averaged potential profile of the convective cell. A scan in bias potential shows that plasma potential modifications are proportional to the electrode current. Therefore, once electron saturation is reached, the plasma potential does not further increase substantially with increasing bias voltage. Using a simple model, we show that such behavior is possible for a sufficiently large level of cross-field currents. We also address quantitatively the question of the origin of these cross-field currents, comparing the importance of currents related with diamagnetic drifts, ion inertia, collisions with neutrals, and viscosity.

This article is organized as follows. Section II discusses the experimental setup and target plasmas. In Sec. III, we elucidate the effects of biasing on the propagation of turbulent structures. This is followed by a detailed analysis of the effect of biasing on time averaged profiles in Sec. IV: Sec. IV A investigates the dependence of the effect of biasing upon the applied bias potential and compares it with simple 1D models; Sec. IV B investigates the toroidal structure of convective cells and Sec. IV C their displacement with respect to the biased flux tube. In Sec. V, we estimate the importance of different sources of cross-field currents and the resulting structure and magnitude of the plasma potential. Results are summarized and discussed in Sec. VI.

\section{EXPERIMENTAL SETUP}

Experiments are conducted in the simple magnetized toroidal device TORPEX, ${ }^{18,19}$ a device dedicated to the basic study of plasma turbulence and transport. Low $\beta$ plasmas are produced and sustained by microwaves in the electron cyclotron range of frequencies ${ }^{20}$ and confined by a dominant toroidal magnetic field $B_{\phi}$ on which a small vertical component $B_{z}$ is superimposed. This leads to helical field lines that wind around the torus and intercept the vacuum vessel at the bottom and the top, as sketched in Figs. 1(a) and 1(b). The dominant instability in TORPEX depends on the value of the vertical field component. By increasing $B_{z}$, a transition from $k_{\|} \neq 0$ modes to modes with $k_{\|}=0$ is observed, ${ }^{21}$ where $k_{\|}$ is the wave number along the magnetic field. Using global, three-dimensional fluid simulations, it has recently been demonstrated that this corresponds to a transition from a turbulent regime dominated by resistive interchange waves to the one dominated by ideal interchange waves. ${ }^{22}$ In the present experiments, we use $B_{\phi} \approx 76 \mathrm{mT}$ and $B_{z} \approx 1.55 \mathrm{mT}$, which falls in the ideal interchange regime. For sufficiently low values of injected microwave power, vertically elongated plasmas are generated. By adjusting the value of $B_{\phi}$, these plasmas can be positioned towards the high-field side (HFS), i.e., the region corresponding to small values of $r$, as defined in Fig. 1(a). The ion saturation current $\left(I_{\text {sat }}\right)$ profile of such a plasma over a cross-section of TORPEX is shown in Fig. 2(a). Hydrogen at a pressure of $\approx 0.02 \mathrm{~Pa}$ is used as working gas and density and electron temperatures are $\lesssim 10^{16} \mathrm{~m}^{-3}$ and $\lesssim 8 \mathrm{eV}$, respectively. The profile of plasma potential $V_{p l}$ and the deduced $\mathbf{E} \times \mathbf{B}$ velocity field is shown in Fig. 2(b). The plasma and heat source are localized at the HFS. The ideal interchange wave develops on the low-field side (LFS) of the profiles and intermittently ejects bunches (a)

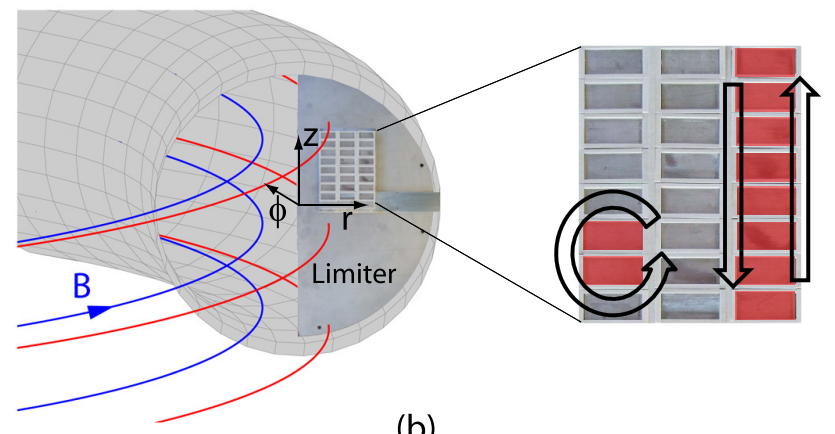

(b)

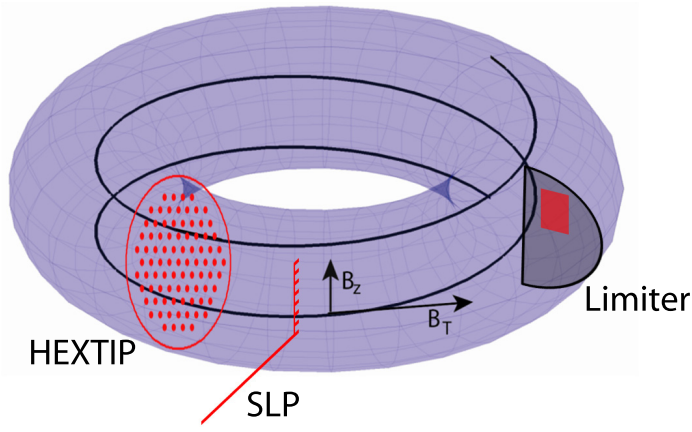

FIG. 1. (a) Sketch of the TORPEX vacuum vessel and the electrodes installed on a conducting limiter. Examples of magnetic field lines and the coordinate system are also shown. The enlarged view of the electrodes shows possible biasing schemes. Shaded areas indicate positively biased electrodes and arrows show the expected flow pattern. (b) Sketch (not to scale) of TORPEX and the LPs used in this work.

of plasma (blobs) radially outwards into a region with negligible plasma production. These blobs are structures of enhanced density that are elongated along the magnetic field but localized in the perpendicular plane. ${ }^{23-25}$ Their formation, ${ }^{26-29}$ subsequent propagation, ${ }^{30-32}$ and associated transport, ${ }^{27,33,34}$ driven by $\nabla B$ and curvature induced polarization, have been extensively studied in this scenario.

For the present experiments, we have installed a grounded stainless steel limiter in the blob propagation region and an array of $3 \times 8$ stainless steel electrodes has been attached on its surface. This is sketched in Fig. 1(a). Each electrode has a rectangular surface of $2 \mathrm{~cm} \times 0.9 \mathrm{~cm}$ and an isolating support. This setup protrudes from the limiter by $\approx 8 \mathrm{~mm}$. Each electrode can be biased individually and its current can be measured. This arrangement allows for some flexibility on the biasing scheme, as shown on the right hand side of Fig. 1(a). Shaded, red areas indicate positively biased electrodes and arrows the expected flow pattern.

Electrodes that are not used for biasing can be used as Langmuir probes (LPs) to measure plasma properties close to the limiter. Additional measurements are taken with a $2 \mathrm{D}$ LP array, dubbed HEXTIP. ${ }^{35}$ This consists of 86 electrodes that cover the whole plasma cross section and provide $I_{s a t}$ or floating potential $V_{f l}$ measurements. HEXTIP is displaced toroidally by $90^{\circ}$ from the limiter, in the clock-wise direction when viewed from above (Fig. 1(b)). Additionally, we use a vertical array of 8 LPs that can be moved radially and rotated in the toroidal direction. This probe, dubbed SLP, is displaced toroidally by $55^{\circ}$ from the limiter and lies thus between limiter and HEXTIP, Fig. 1(b). Besides measuring 


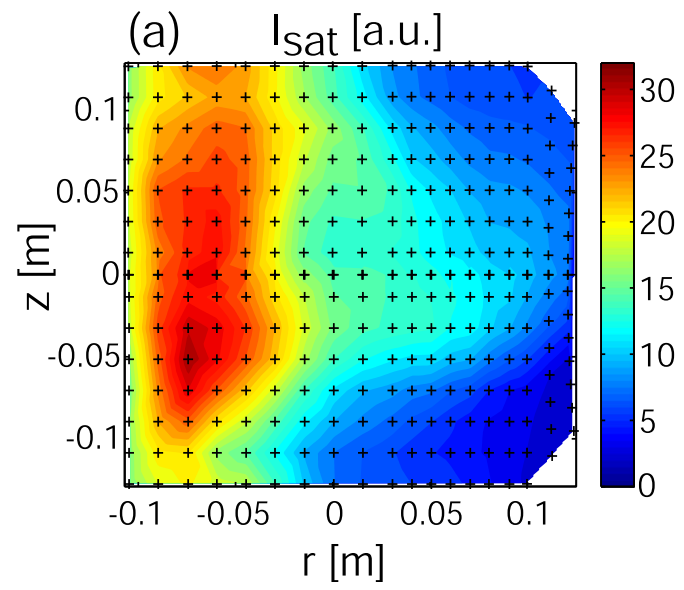

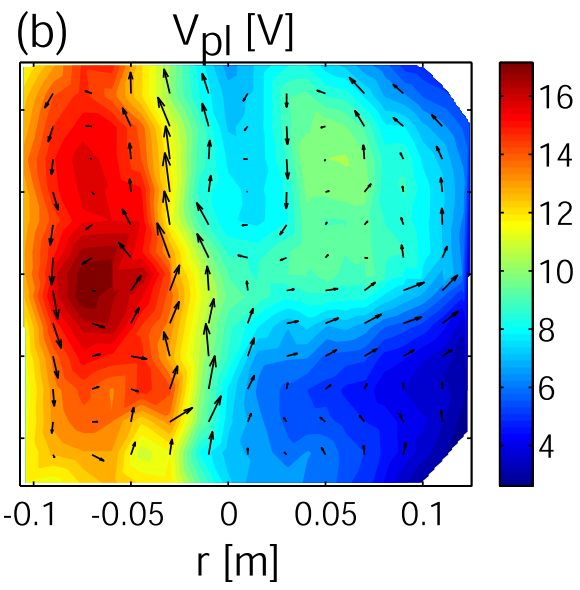

FIG. 2. Profiles of (a) $I_{s a t}$ and (b) $V_{p l}$ measured with SLP. Crosses in (a) indicate the measurement points and vectors in (b) the $\mathbf{E} \times \mathbf{B}$ velocity field deduced from $V_{p l}$.
$I_{s a t}$ or $V_{f l}$, it can be operated in swept mode to measure the time averaged I-V characteristics.

\section{EFFECT OF BIASING ON BLOBS}

In this section, we present two examples that show how biasing affects blob propagation. ${ }^{17}$ In the first example, we have selected a vertical stripe of 8 biasing electrodes. In a first phase of the discharge, the "bias on" phase, these electrodes are biased to $+40 \mathrm{~V}$. During a second phase, the "bias off" phase, they are grounded (the other 16 electrodes are grounded during both phases). To see the effect of this on blob propagation, we perform conditional average sampling (CAS): ${ }^{28,29,36}$ A HEXTIP tip in the blob region is used as reference probe to select local maxima in the $I_{\text {sat }}$ time trace exceeding 2.5 times the signal standard deviation. These events are interpreted as blobs passing in front of the probe. The average value of $I_{\text {sat }}$ on an SLP probe for a given time lag with respect to the selected events is then evaluated. This is done for different time lags, for all eight probe signals from SLP and for different radial positions of SLP (moving it in between highly reproducible discharges). This then allows reconstructing the 2D, conditionally averaged blob propagation in the SLP plane.

Successive time frames obtained with this procedure are shown in Fig. 3. Time $t=0$ corresponds to the time when the blobs are detected on the reference probe. Color plots show results for the bias on phase, while the $I_{\text {sat }}$ contours of the blob during the bias off phase are shown in white. The set of electrodes used for the biasing is indicated at their field line mapped position in the SLP plane by black rectangles. While early in time, blob propagation is similar in the bias on and off phases, clear differences are apparent later on. As anticipated, the blob is strongly pushed downwards due to the biased electrodes. In the time interval $[-8 \mu \mathrm{s}, 8 \mu \mathrm{s}]$, the blob vertical velocity changes due to the biasing from $v_{z} \approx-700 \mathrm{~ms}^{-1}$ to $v_{z} \approx-2100 \mathrm{~ms}^{-1}$. The reduction in time of the blob amplitude, which is also apparent from Fig. 3 is a general feature of CAS (Ref. 28) and is also observed in the absence of biasing.
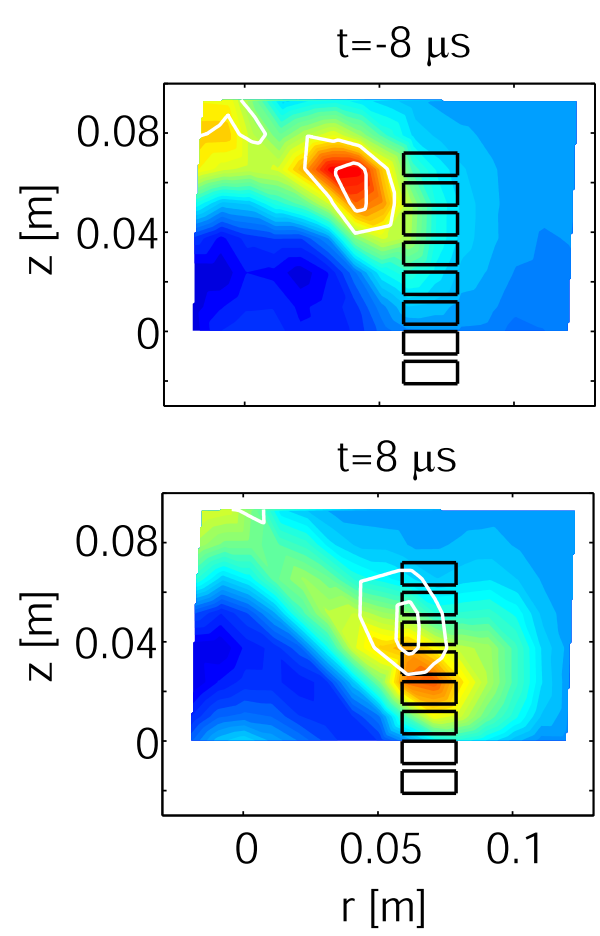
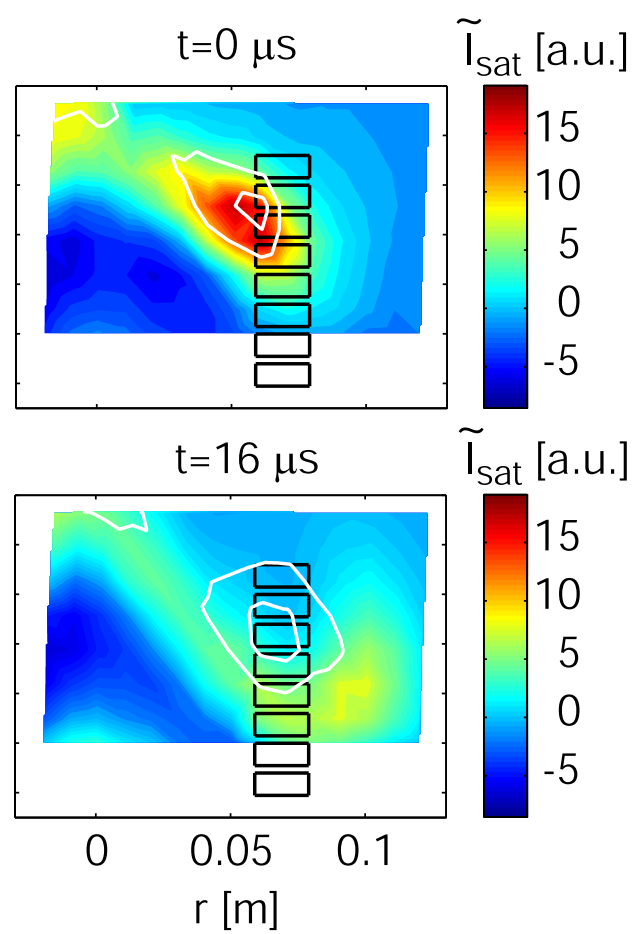

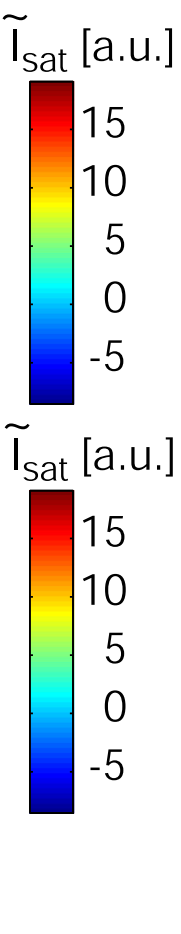

FIG. 3. Conditionally averaged blob propagation ( $I_{\text {sat }}$ fluctuations) for the case where a vertical stripe of electrodes is biased to $+40 \mathrm{~V}$ (color plots). For comparison, the white contours indicate the results of the same analysis when all electrodes are grounded (Reprinted with permission from C. Theiler, I. Furno, J. Loizu, and A. Fasoli, Phys. Rev. Lett. 108, 065005 (2012). Copyright (C) (2012) American Physical Society). 

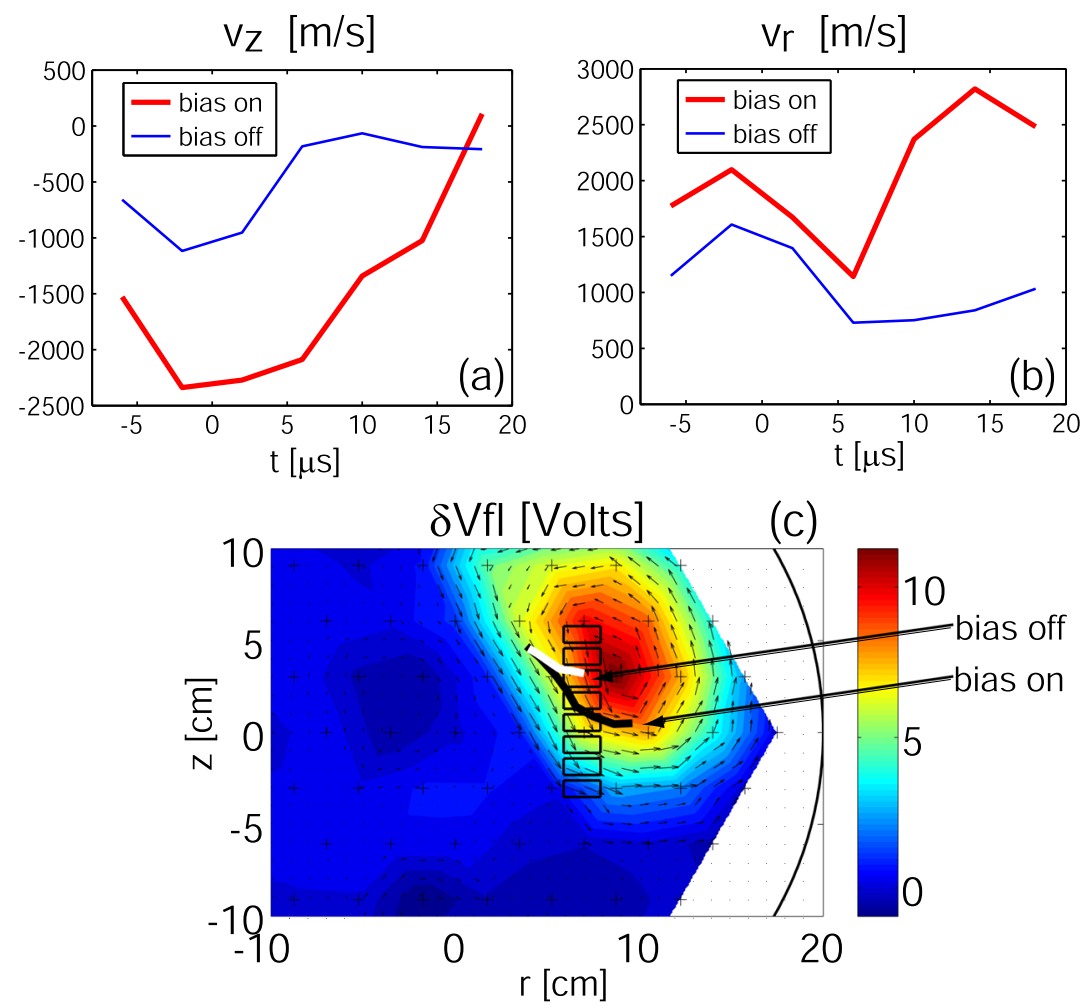

FIG. 4. (a) and (b) Vertical and radial velocity of the conditionally averaged blob propagation of Fig. 3 . (c) Change in time-averaged floating potential induced by the biasing. The blob trajectories in the measurement plane are also shown. (d) and (e) Profiles of changes in the $\mathbf{E} \times \mathbf{B}$ flow, deduced from $\delta V_{f l}$.
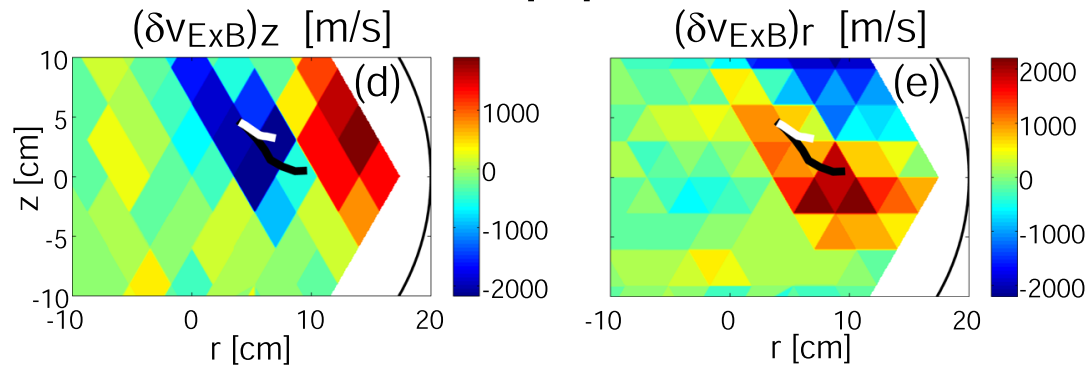

Fig. 4 illustrates the changes in blob velocity in more detail and shows a comparison with measured changes in floating potential and deduced $\mathbf{E} \times \mathbf{B}$ flows. Figs. 4(a) and 4(b) show the blob vertical and radial velocity as a function of time for the bias off (thin blue) and the bias on cases (thick red). These values are obtained from the center of mass position of the conditionally averaged blob, evaluated for each time frame. In Fig. 4(c), we show the measured change $\delta V_{f l}$ of the timeaveraged floating potential profile caused by the biasing. $\delta V_{f l}$ is measured with HEXTIP and linearly interpolated between the measurement points. Also shown is the field-line mapped position of the biased electrodes (black rectangles) and the trajectory of the blob for the bias off (white) and the bias on (black) phases. The black vectors indicate the perturbations of the $\mathbf{E} \times \mathbf{B}$ flow, dubbed $\delta \mathbf{v}_{\mathbf{E} \times \mathbf{B}}$, deduced from $\delta V_{f l}$. Qualitatively, we see that the deviation of the trajectory for the biased blob occurs in the direction of $\delta \mathbf{v}_{\mathbf{E} \times \mathbf{B}}$.

To be more quantitative, we plot in Figs. 4(d) and 4(e) the vertical and radial components of $\delta \mathbf{v}_{\mathbf{E} \times \mathbf{B}}$. For $t \in[-8 \mu \mathrm{s}$, $12 \mu \mathrm{s}]$, the biased blob passes through a region where $\left(\delta \mathbf{V}_{\mathbf{E} \times \mathbf{B}}\right)_{z}$ is strongly negative (between $-1400 \mathrm{~ms}^{-1}$ and $-2200 \mathrm{~ms}^{-1}$ ). This is in good agreement with the observed difference in vertical velocity in Fig. 4(a) between the biased and the unbiased blobs. In the interval $[12 \mu \mathrm{s}, 20 \mu \mathrm{s}]$, the biased blob passes through a region with a large radial flow perturbation, $\left(\delta \mathbf{v}_{\mathbf{E} \times \mathbf{B}}\right)_{r} \approx 1900 \mathrm{~ms}^{-1}$. Again, this is in good agreement with the observed difference in radial velocity in Fig. 4(b) between the biased and the unbiased blobs.

In Fig. 5, we show another example where blob velocity is successfully modified. A set of four electrodes are used to induce a counter-clockwise rotating cell. As expected, blobs passing below this set of electrodes are radially accelerated with respect to the unbiased case. In the time interval [ $8 \mu \mathrm{s}, 16 \mu \mathrm{s}]$, the blob radial velocity increases due to the biasing from $v_{r} \approx 1200 \mathrm{~ms}^{-1}$ to $v_{r} \approx 2200 \mathrm{~ms}^{-1}$. An analysis similar to the one shown in Fig. 4 also shows good agreement between changes in blob velocity and values of $\delta \mathbf{v}_{\mathbf{E} \times \mathbf{B}}$ deduced from $\delta V_{f l}$.

These results demonstrate that biasing allows changing both radial and vertical blob velocities. Velocity changes are in quantitative agreement with the convective motion deduced from the measured profile of $\delta V_{f l}$. As apparent from Fig. 4(c), the latter can, however, differ from what one would expect from the bias configuration. In Secs. IV and V, the physics determining the structure of $\delta V_{f l}$ is investigated in detail.

We note that even in the unbiased case in Fig. 2(b)), a structure in $V_{p l}$ is observed at the LFS. This could indicate radial steady state flows as reported also from tokamaks. ${ }^{37}$ Origin and possible contributions of this to blob motion on TORPEX is not understood. ${ }^{38}$ 

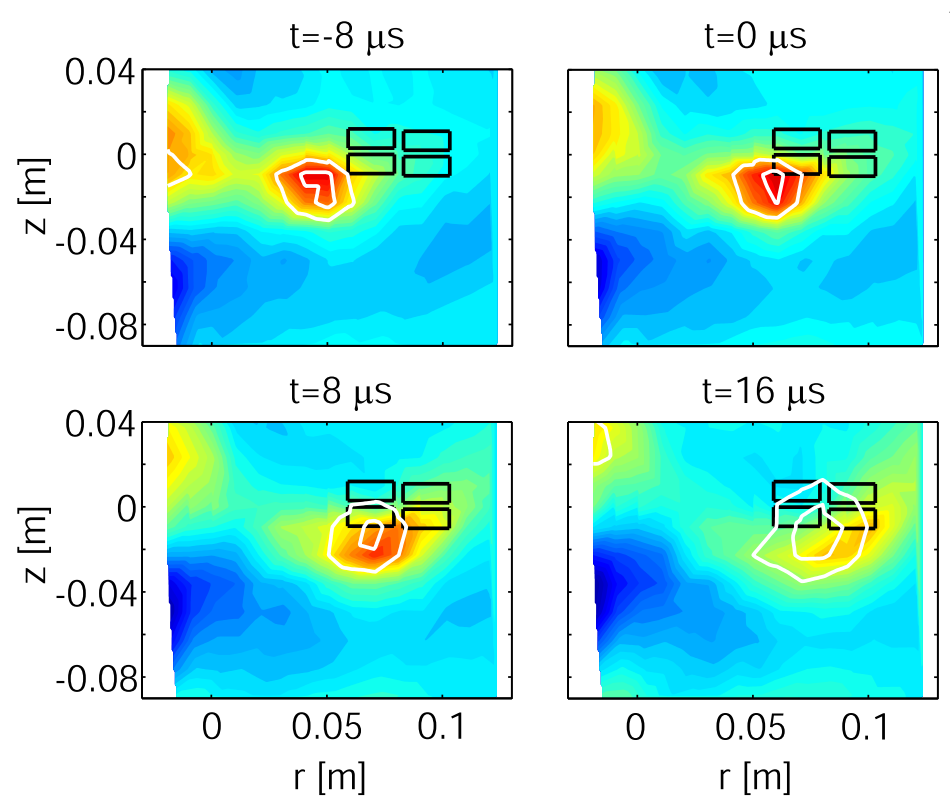

\section{TIME AVERAGED PROPERTIES OF BIASING}

We focus now on the effect of biasing on time averaged profiles. On TORPEX, plasma potential measurements are obtained from $V_{p l}=V_{f l}+\mu T_{e}$, where $\mu \approx 3,{ }^{20,39}$ and, due to the open field lines, plasma potential is mainly determined by the temperature profile. Despite this, we identify variations in plasma potential, $\delta V_{p l}$, due to the biased electrodes with variations in floating potential. This approach is justified by the observation that biasing in our experiments has more effect on the floating potential than on electron temperature, such that $\delta V_{p l}=\delta V_{f l}+\mu \delta T_{e} \approx \delta V_{f l}$, as shown by the following test. We have operated SLP in sweep mode to reconstruct the quantities $n, T_{e}, V_{f l}$, and $V_{p l}$ during a bias on and a bias off phase. During the bias on phase, a pair of electrodes is biased to $+40 \mathrm{~V}$. 2D profiles of plasma parameters are obtained by moving SLP in between discharges. In Figs. 6(a) and 6(b), we compare the measured $\delta V_{p l}$ and $\delta V_{f l}$ obtained in this way. We observe a similar structure of potential variations with a peak shifted with respect to the biased flux tube in both cases. The two profiles also agree rather well quantitatively, thus $\delta V_{f l} \approx \delta V_{p l}$. Fig. 6(c) shows the measurement of $\delta V_{f l}$ obtained with HEXTIP. This reveals a satisfactory agreement with Figs. 6(a) and 6(b), despite a lower spatial resolution, as indicated by the black dots. The measurements of $\delta I_{\text {sat }} / I_{\text {sat }}$ obtained with the two diagnostics also give a similar picture, Figs. 6(d) and 6(e).

The measurements in Figs. 6(a) and 6(d) reveal characteristics of a convective cell, namely a positive structure in $\delta V_{p l}$ (and $\delta V_{f l}$ ) and changes in $I_{s a t}$ up to $60 \%$. These are consistent with an outward convection at the bottom and an inward convection at the top of the $\delta V_{p l}$ structure. What might have been less expected is the relatively large size of the $\delta V_{p l}$ structure and its vertical and radial displacement with respect to the biased flux tube. Further, the peak value of $\delta V_{p l}$ of $\approx 4 \mathrm{~V}$, which corresponds to about 1.8 times the electron temperature in that region, is well below the applied bias of $+40 \mathrm{~V}$. Structure and properties of the observed convective cells are further explored in the following.

\section{A. 1D versus 2D}

To investigate more closely the limitation on the achievable plasma potential modifications, we look at a series of discharges with different values of bias potential. Figs. 7(a)-7(c) show the measured potential variations for bias voltages of $-40 \mathrm{~V},+3 \mathrm{~V}$, and $+40 \mathrm{~V}$, applied to the pair of electrodes indicated as black rectangles at their field-line mapped position in the HEXTIP plane. For the strong negative bias, negative values of $\delta V_{f l}$ around the biased flux tube are of low amplitude. For $+3 \mathrm{~V}$, a positive structure of $\delta V_{f l}$, similar to the one in Fig. 6(c), is apparent. This increases in amplitude while keeping a similar structure as the applied bias voltage is gradually increased. In Fig. 7(d), we plot the electrode current for the different bias voltages (here, the intermediate values $+6 \mathrm{~V}$, $+9 \mathrm{~V}$, and $+12 \mathrm{~V}$ are also included). This shows a strong asymmetry in the electrode current for positive and negative bias voltages. The horizontal, dashed line shows $\sqrt{2 m_{i} /\left(\pi m_{e}\right)} \cdot\left|I_{\text {sat }}\right| \approx 34 \cdot\left|I_{\text {sat }}\right|$, evaluated for atomic hydrogen. This is the value expected for the electron saturation current of an ideal LP. ${ }^{40}$ The measured currents for strong positive bias are close to this value.

These observations are qualitatively similar to the results from NSTX, where the electrode current at large positive bias exceeds the ion saturation current by a factor $\approx 8 .{ }^{15}$ In the MAST experiments, on the contrary, this factor was $\approx 1 .^{13}$ This was expected, however, as the ratio of grounded to biased surfaces was at most equal to 3 (taking into account grounded ribs of both the upper and the lower divertors). ${ }^{41,42}$

In Fig. 7(d), we also plot the peak value of $\delta V_{f l}$ for the different bias voltages (dashed green curve). It essentially falls on top of the current curve and is thus proportional to the electrode current. Such a dependence was also observed in the divertor electrode biasing experiments in NSTX for large electrode currents. ${ }^{16}$ As the electrode current is limited by the electron saturation current, this sets a limit on the potential that can be induced in these plasmas. 

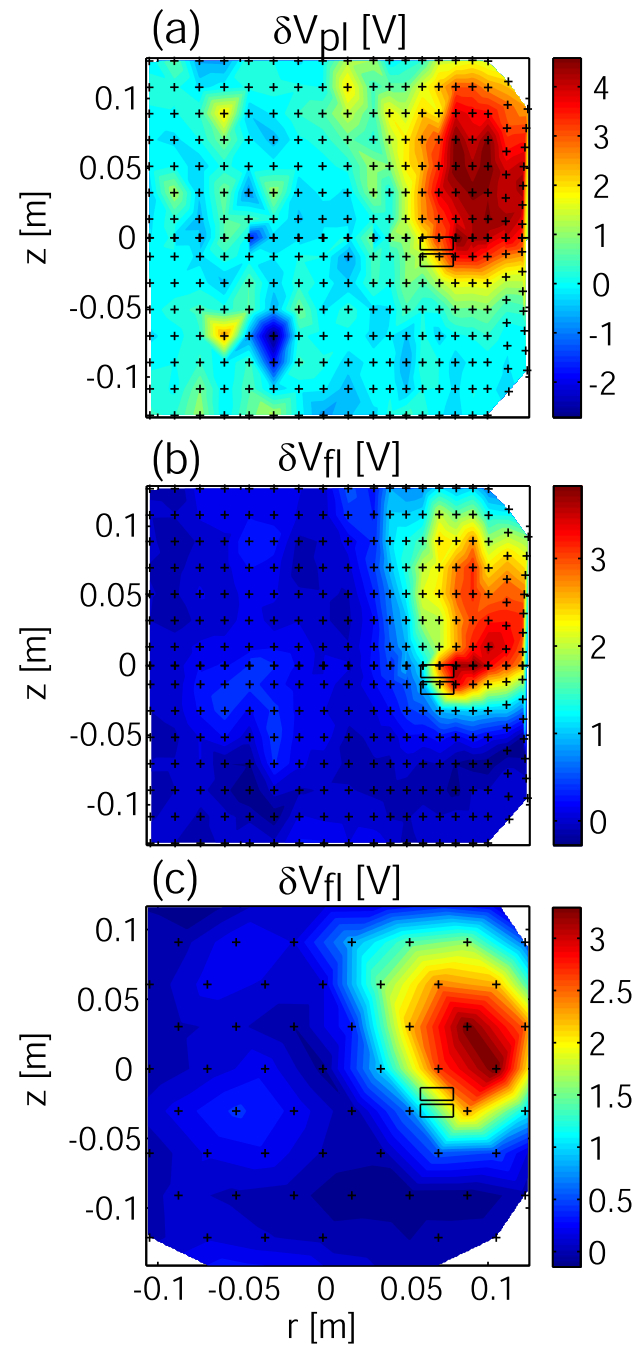

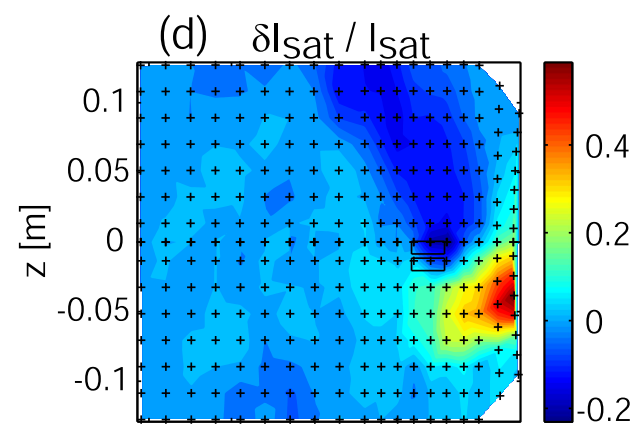

(e) $\delta \mathrm{I}_{\text {sat }} / \mathrm{I}_{\text {sat }}$

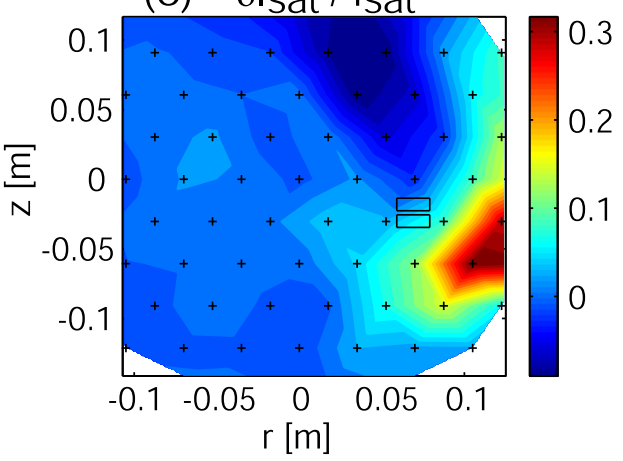

FIG. 6. Tests performed to justify the use of $V_{f l}$ measurements to indicate plasma potential variations induced by the biasing and the limited spatial resolution of the HEXTIP probe. Plots (a), (b), and (d) are obtained from the SLP probe operated in swept mode and moved in between reproducible discharges. (c) and (e) are measurements with HEXTIP. Black dots indicate the measurement positions. A slightly different range of the vertical axis for HEXTIP and SLP accounts for the pitch of the magnetic field.
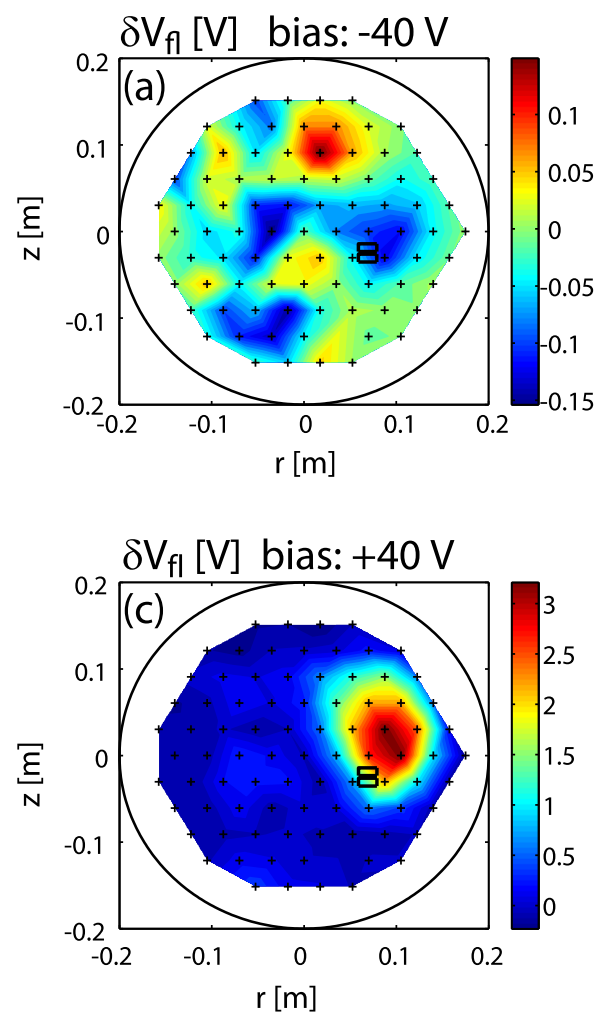
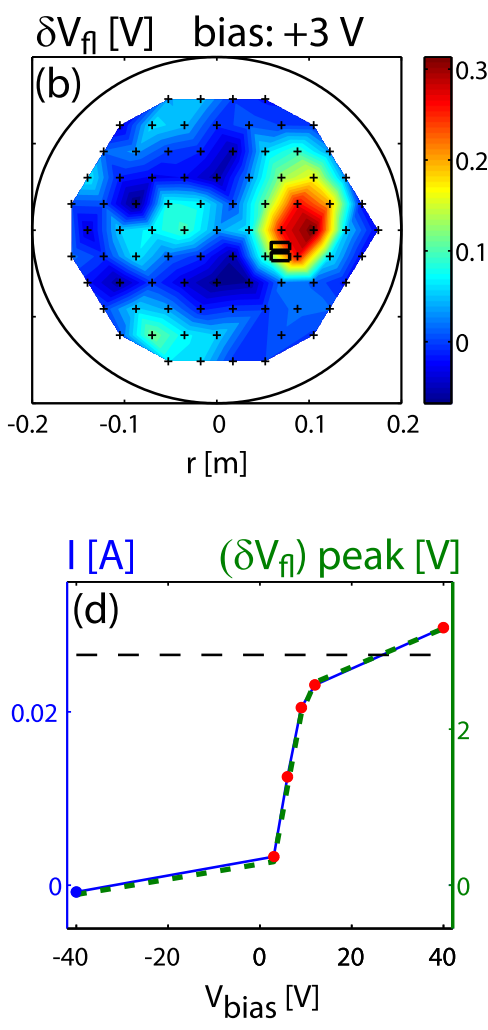

FIG. 7. (a)-(c) Potential modifications induced by different values of bias potential. (d) Current on the two biased electrodes (left axis, blue solid curve) and resulting potential modifications in the plasma (right axis, green dashed curve) for six different values of bias potential. The black dashed line indicates the expected level of the electron saturation current, obtained by multiplying the probe current at $-40 \mathrm{~V}$ by $-\sqrt{2 m_{i} /\left(\pi m_{e}\right)}$. 
We now compare our results with the quasi 1D model introduced in Refs. 41 and 42. We assume an isothermal, frictionless plasma with a simplified expression for sheath currents given by

$$
j_{\|}=n_{s e} c_{s} e\left(1-\exp ^{\frac{-e\left(V_{p l}-\mu T_{e}-V_{\text {wall }}\right)}{T_{e}}}\right),
$$

as long as $j_{\|}>-\alpha_{r} n_{s e} c_{s} e$ and

$$
j_{\|}=-\alpha_{r} n_{s e} c_{s} e
$$

otherwise, i.e., we assume here a perfect saturation of the electron saturation current. The term $\mu T_{e}$ is the floating value of the plasma potential, ${ }^{40} V_{\text {wall }}$ the potential of the wall or the electrode, $n_{s e}$ the sheath edge density, and $\alpha_{r}$ $=\sqrt{2 m_{i} /\left(\pi m_{e}\right)} \approx 34$ the assumed ratio of electron to ion saturation current. We consider a flux tube in contact with the biased electrode at one end and a grounded, conducting surface at the other end. If currents perfectly follow field lines, the electrode current closes over a grounded surface of equal area. In the presence of cross-field currents, however, currents can be collected over a significantly larger grounded surface area. The quasi 1D model takes this into account. It defines $A$ as the ratio of the current collection areas of the grounded surface to that of the electrode. Electrode current and plasma potential variations as a function of bias potential are then obtained from the current continuity condition

$$
j_{\|}\left(V_{\text {wall }}=V_{\text {bias }}\right)+A \cdot j_{\|}\left(V_{\text {wall }}=0\right)=0 .
$$

Results for different values of A are plotted in Fig. 8. The case $A=1$ is the 1D situation where no cross-field currents exist. The electrode current never exceeds ion saturation current and $\delta V_{p l}$ is roughly proportional to $V_{\text {bias }}$ for positive bias. For finite cross-field currents $(A>1)$, the currentvoltage characteristics become asymmetric while $\delta V_{p l}$ still increases strongly with $V_{\text {bias }}$. Only when $A>\alpha_{r}$ does $\delta V_{p l}$ saturate for large values of $V_{\text {bias }}$. This behavior is what is seen in the experiment, Fig. 7, and highlights the large level of cross-field currents.

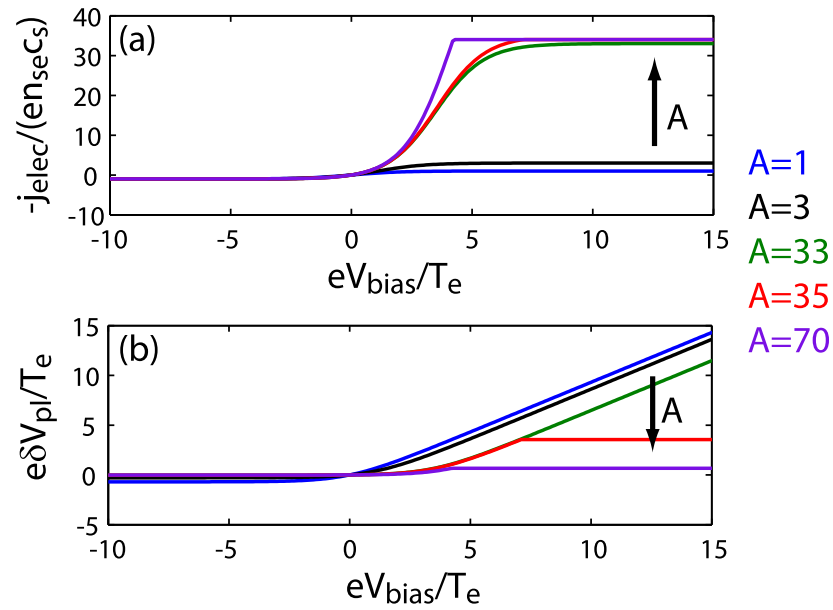

FIG. 8. Normalized (a) electrode current and (b) plasma potential variation as a function of bias potential for the quasi $1 \mathrm{D}$ model, Eq. (3). ${ }^{41,42}$ Different values of $A$, the ratio of current collecting surface to probe surface, has been assumed.

\section{B. 2D versus $3 \mathrm{D}$}

In this section, we investigate the 3D structure of convective cells, i.e., we investigate how modifications of the $V_{f l}$ and $I_{\text {sat }}$ profiles induced by the biasing depend on the toroidal position. To this end, measurements directly on the limiter are compared with measurements across the same flux tube in the HEXTIP plane, toroidally separated by $90^{\circ}$. This set-up is sketched in Fig. 9(g). During the bias on phase, we apply a voltage of $+40 \mathrm{~V}$ to a pair of electrodes and ground them during the bias off phase. The other 22 electrodes are grounded except for one of them, which is used as a wall probe, operated either in $I_{s a t}$ or $V_{f l}$ mode. During a series of reproducible discharges, each of these 22 electrodes is alternately used as wall probe. The 2D measurements of $\delta V_{f l}$ and $\delta I_{\text {sat }} / I_{\text {sat }}$ in the limiter plane obtained this way are plotted in the right column of Fig. 9. In the left column, we plot

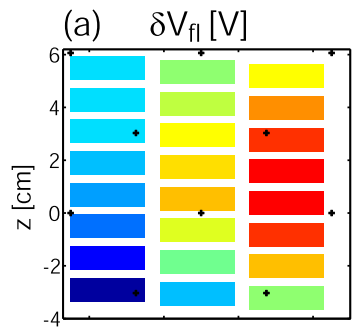

(c) $\delta \mathrm{I}_{\text {sat }} / \mathrm{I}_{\mathrm{sat}}$
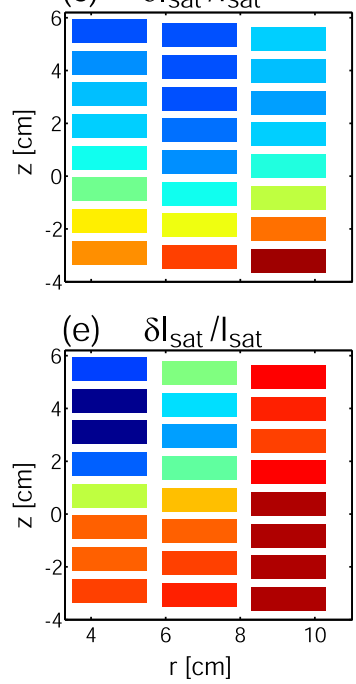

(b) $\delta \mathrm{V}_{\mathrm{fl}}[\mathrm{V}]$

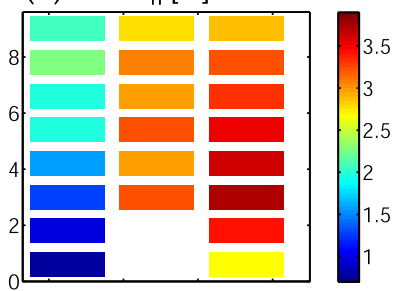

(d) $\delta I_{\text {sat }} / I_{\text {sat }}$
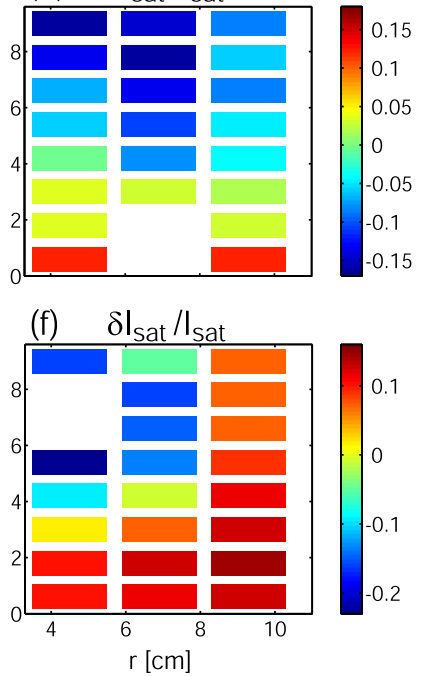

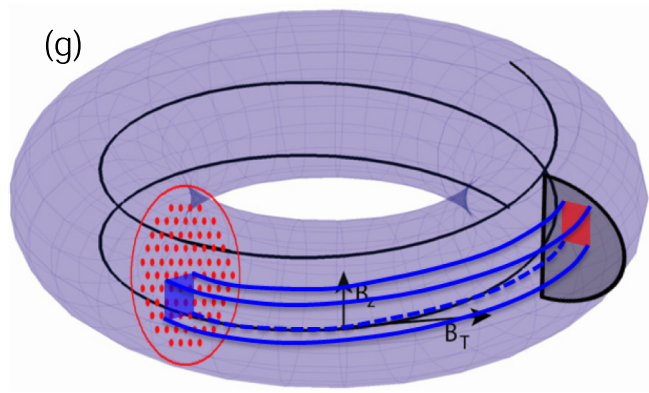

FIG. 9. (a), (c), and (e) Effects on $V_{f l}$ and $I_{\text {sat }}$ profiles due to the biasing, measured with HEXTIP, at $90^{\circ}$ from the limiter. Measurements at the positions indicated by black dots in (a) are linearly interpolated to obtain the values on the flux tubes connected to each limiter electrode. (b), (d), and (f) The same measurements, but obtained directly on the limiter. No measurements are available at the position of the electrodes that are used for the biasing. (g) Sketch of the measurement setup. 
the corresponding measurements in the HEXTIP plane. The measurements from the HEXTIP probe tips at the positions indicated by black dots in Fig. 9(a) are linearly interpolated to obtain the values on the flux tubes connected to each electrode.

Measurements of $\delta V_{f l}$, Figs. 9(a) and 9(b), do not reveal strong differences in structure and absolute values in the two toroidally separated planes. In both cases, the $\delta V_{f l}$ structure is shifted radially and vertically with respect to the biased electrodes. Further, we find $\delta V_{f l} \lesssim 4 \mathrm{~V}$ in both planes. Good agreement is also found for relative changes in $I_{\text {sat }}$, Figs. 9(c) and $9(\mathrm{~d})$. These $I_{\text {sat }}$ measurements are repeated with the bias applied to a different set of electrodes, Figs. 9(e) and 9(f). In this case, a larger fraction of the region, where $I_{\text {sat }}$ increases due to the bias is captured. Again, we find good agreement between the two planes.

These measurements suggest that changes induced by the biasing are not strongly dependent on the toroidal direction and that the problem is fairly $2 \mathrm{D}$, although measurements directly in front of the biased electrodes are not available and we can thus not exclude significant 3D effects in this region.

\section{The effect of convective motion}

As we have clearly seen, e.g., in Fig. 6(a), the $\delta V_{p l}$ structure is not centered around the flux tube where the bias is applied, but shifted both upwards and radially outwards. In this section, we investigate the reason for this non-locality of the effect of biasing.

Comparing the $\mathbf{E} \times \mathbf{B}$ velocity field in Fig. 2(b) with the $\delta V_{p l}$ profile in Fig. 6(a) suggests that $\delta V_{p l}$ is shifted in the direction of plasma flows. This hypothesis is tested here with a series of measurements with reversed direction of the mag- netic, i.e., where we set $B_{\phi} \rightarrow-B_{\phi}$ and $B_{z} \rightarrow-B_{z}$. In this case, the steady-state vertical $\mathbf{E} \times \mathbf{B}$ flow is directed downwards at the LFS and upwards at the HFS of the $I_{\text {sat }}$ profile. Fig. 10(a) shows $\delta V_{f l}$ for a bias applied in the blob region. The location of the $I_{\text {sat }}$ profile is indicated by white contours. Consistent with a vertical inversion of the flow pattern, $\delta V_{f l}$ is now shifted downwards and radially outwards. In Fig. 10(b), the whole plasma is displaced radially outwards by increasing the magnitude of $B_{\phi}$. Biasing is thus applied to a region close to the peak of the $I_{\text {sat }}$ profile. In this region, no large flows are present and we find that $\delta V_{f l}$ is well centered around the biased flux tube. If we increase $\left|B_{\phi}\right|$ further, Fig. 10(c), the bias is applied to the HFS region of the $I_{\text {sat }}$ profile, where the $\mathbf{E} \times \mathbf{B}$ flow is directed upwards. In this case, the $\delta V_{f l}$ profile is shifted upwards as well. These measurements confirm the hypothesis that the observed displacement of the $\delta V_{p l}$ profile with respect to the biased flux tube is caused by plasma flows. Further support for this is given in Sec. V B.

\section{ESTIMATES OF CROSS-FIELD CURRENTS}

The biasing experiments presented in Secs. III and IV have demonstrated clear effects of biasing on blob propagation and time averaged profiles. At the same time, rather important limitations on the magnitude of the achievable potential variations have been observed. These are due to cross-field currents in the plasma, as discussed in Sec. IV A. In the following, we address the question of the origin of these currents.

As discussed, e.g., in Ref. 43, cross-field currents in magnetized plasmas can mainly be attributed to diamagnetic drifts, ion-polarization, collisions with neutrals, and viscosity. We write the cross-field current $\mathbf{j}_{\perp}$ as a sum of these contributions
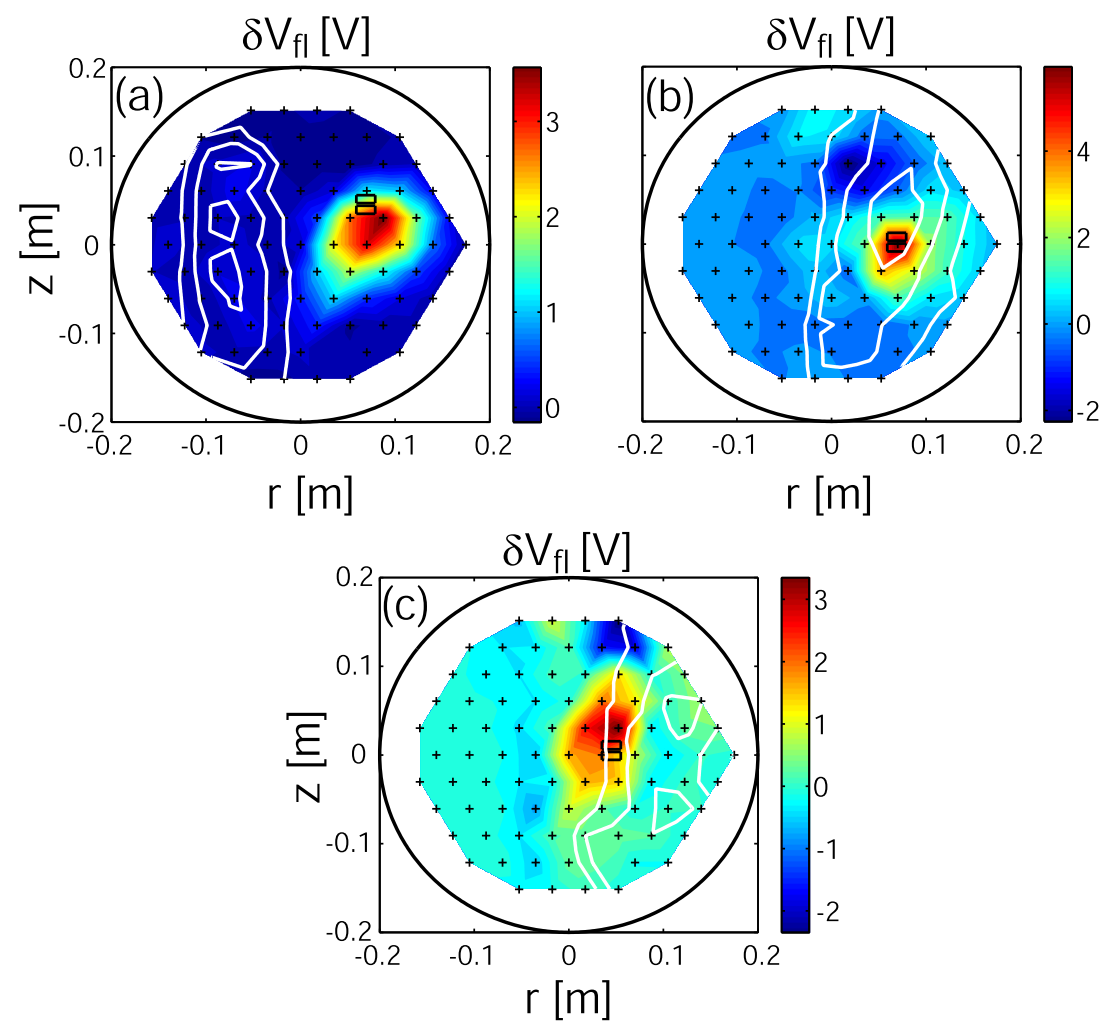

FIG. 10. $\delta V_{f l}$ measured in plasmas with reversed field $\left(B_{\phi}, B_{z}<0\right)$ and different values of $\left|B_{\phi}\right| . I_{\text {sat }}$ profiles are indicated by white contours. 


$$
\mathbf{j}_{\perp}=\mathbf{j}_{\perp}^{\text {dia }}+\mathbf{j}_{\perp}^{\text {pol }}+\mathbf{j}_{\perp}^{\mathrm{i} / \mathrm{n}}+\mathbf{j}_{\perp}^{\mathrm{e} / \mathrm{n}}+\mathbf{j}_{\perp}^{\mathrm{visc}}
$$

where the superscripts $i / n$ and $e / n$ indicate currents related to ion-neutral and electron-neutral collisions, respectively. Current continuity, $\nabla \cdot\left(\mathbf{j}_{\perp}+\mathbf{j}_{\|}\right)=0$, averaged along the magnetic field lines, gives

$$
\left\langle\nabla_{\perp} \cdot \mathbf{j}_{\perp}\right\rangle=-\frac{1}{L_{c}} j_{\| \text {tot }},
$$

where brackets indicate an average along the magnetic field lines of length $L_{c}$ and $j_{\| \text {tot }}$ is the total current density flowing out of the flux tube at both ends. Below, we derive equations for the equilibrium plasma potential from Eq. (5) for different mechanisms of cross-field currents. In order to do this, we evaluate the divergence of $\mathbf{j}_{\perp}$. Based on the drift-reduced Braginskii equations ${ }^{44}$ in the electrostatic limit, ${ }^{45}$ we find

$$
\begin{gathered}
\nabla \cdot \mathbf{j}_{\perp}^{\mathrm{dia}}=\frac{2}{B R} \partial_{z}\left(p_{i}+p_{e}\right), \\
\nabla \cdot \mathbf{j}_{\perp}^{\mathrm{pol}}=\nabla \cdot\left[\frac{n_{0} m_{i}}{B^{2}} \frac{\mathrm{d}}{\mathrm{d} t}\left(\mathbf{E}_{\perp}-\frac{\nabla_{\perp} p_{i}}{e n_{0}}\right)\right], \\
\nabla \cdot \mathbf{j}_{\perp}^{\mathrm{i} / \mathrm{n}}=\nabla \cdot\left[\frac{n_{0} m_{i}}{B^{2}} \nu_{i n}\left(\mathbf{E}_{\perp}-\frac{\nabla_{\perp} p_{i}}{e n_{0}}\right)\right], \\
\nabla \cdot \mathbf{j}_{\perp}^{\mathrm{e} / \mathrm{n}}=\nabla \cdot\left[\frac{n_{0} m_{i}}{B^{2}} \frac{m_{e}}{m_{i}} \nu_{e n}\left(\mathbf{E}_{\perp}+\frac{\nabla_{\perp} p_{e}}{e n_{0}}\right)\right], \\
\nabla \cdot \mathbf{j}_{\perp}^{\mathrm{visc}}=-\frac{\eta_{0}}{3 B^{2} R^{2}}\left(2 B \partial_{z} \partial_{\phi} V_{\| i}+\partial_{z}^{2} V_{p l}\right. \\
\left.+\frac{1}{e n_{0}} \partial_{z}^{2} p_{i}-\frac{1}{e n_{0}^{2}} \partial_{z} n_{0} \partial_{z} p_{i}\right)+\frac{\eta_{1}}{B^{2}} \Delta\left(\Delta V_{p l}\right) .
\end{gathered}
$$

Here, $\nu_{i n}$ and $\nu_{e n}$ are the ion-neutral and electron-neutral collision frequencies, $\frac{\mathrm{d}}{\mathrm{d} t}=\partial_{t}+v_{\mathbf{E} \times \mathbf{B}} \cdot \nabla_{\perp}$ the convective derivative, $v_{\mathbf{E} \times \mathbf{B}}$ the $\mathbf{E} \times \mathbf{B}$ drift, $V_{\| i}$ the parallel ion velocity, $n_{0}$ the density in the bulk plasma, $p_{i}$ and $p_{e}$ the ion and electron pressure, and $\eta_{0}=0.96 n_{0} T_{i} \tau_{i}$ and $\eta_{1}=0.3 n_{0} T_{i} /\left(\omega_{c i}^{2} \tau_{i}\right)$ the viscosity coefficients. The coordinate system defined in Fig. 1(a) is used. In the derivation of the above expressions, we have assumed constant values of $\eta_{0}$ and $\eta_{1}$ and small angles between the gradients of $n_{0}$ and $T_{i}$.

To estimate the importance of the different cross-field current terms, we use Eqs. (6)-(10) assuming the following param- eters: $n_{0}=10^{16} \mathrm{~m}^{-3}, T_{e}=2.5 \mathrm{eV}, T_{i} \in[0.1,1] \mathrm{eV}, R=1 \mathrm{~m}$, $B=0.076 \mathrm{~T}, v_{\mathbf{E} \times \mathbf{B}}=1000 \mathrm{~ms}^{-1}$, a perpendicular and parallel scale length of $1 \mathrm{~cm}$ and $3 \mathrm{~m}$, respectively, $m_{i}=1 \mathrm{amu}$, $\nu_{i n}=5 \cdot 10^{4} \mathrm{~s}^{-1}$, and $\nu_{e n}=10^{6} \mathrm{~s}^{-1}$. We then find ratios for the magnitude of the different current sources, Eqs. (6)-(10), of 11:2:1:0.04:0.003 (for $T_{i}=0.1 \mathrm{eV}$ ), and $15: 3: 1.5: 0.04: 1$ (for $T_{i}=1 \mathrm{eV}$ ).

This suggests that diamagnetic currents are the dominant source for cross-field currents. However, from Eqs. (5) and (6), we see that the vertical derivative of the pressure profile would need to become more positive with bias when diamagnetic currents were to balance the negative electrode current. Looking, e.g., at Fig. 6(d) suggests that rather the opposite is observed. In the following, we therefore investigate in more detail the next two candidates, that is, currents due to ionneutral collisions and ion-polarization currents.

\section{A. Currents related with ion-neutral collisions}

To estimate the importance of cross-field currents related with ion-neutral collisions, we consider a situation with a circular electrode of radius $a$ installed on a conducting limiter, as sketched in Fig. 11(a). We assume that the magnetic field lines intercept the limiter perpendicularly and they end on another conducting plate located at a distance $L_{c}$. In the experiments, this second plate corresponds to the backside of the limiter. Such scenarios have been investigated theoretically to model flush mounted Langmuir probes ${ }^{43,46,47}$ and here, we adopt a similar but simplified approach.

Motivated by the measurements discussed in Sec. IV B, we assume no variations along the magnetic field. For simplicity, we further assume uniform ion temperature and plasma density, except for the boundary condition of parallel currents, where we allow for a pre-sheath density drop $n_{s e} / n_{0} \neq 1$. Retaining only the contribution of ion-neutral collisions in $\mathbf{j}_{\perp}$, Eq. (5) then gives

$$
\Delta_{\perp} V_{p l}=\frac{B^{2}}{n_{0} m_{i} L_{c} \nu_{i n}} j_{\| \text {tot }} .
$$

We assume large bias voltages such that the electrode draws electron saturation current over its whole surface. To maintain quasi-neutrality, the loss of electrons is then compensated by ions flowing out perpendicularly to the biased flux tube. The associated particle loss is assumed to be compensated by ambipolar cross-field transport.
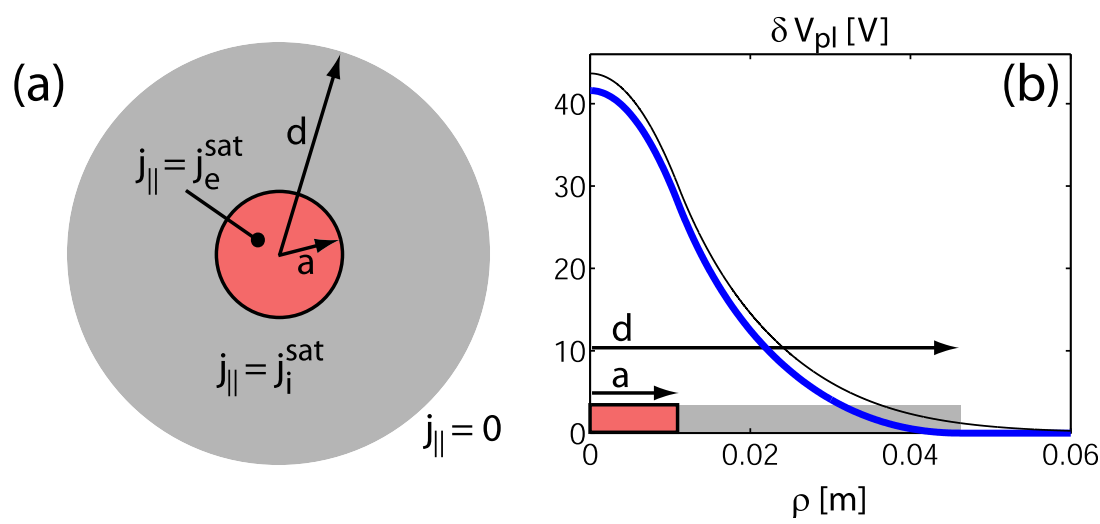

FIG. 11. Calculation of the expected plasma potential variations induced by a circular, positively biased electrode in a steady-state plasma where ion-neutral collisions are the only source of cross-field currents. The thick blue curve in (b) is obtained from solving Eq. (11), assuming a simplified structure of the parallel current density, as sketched in (a). For the thin black curve, Eqs. (1) and (2) are assumed instead for the parallel current density. 
In a first step, we take a simplified structure for the parallel current. We assume that the electron saturation current drawn by the electrode closes by ion saturation currents flowing to the grounded parts of the limiter at both ends of a flux tube of radius $d$. No parallel currents exist outside this radius. To assure charge conservation, the radius $d$ is given by $d=\sqrt{\left(\alpha_{r}+1\right) / 2} \cdot a$, where, as in Sec. IV A, $\alpha_{r}$ is the ratio of electron and ion saturation current. A sketch of this current structure on and around the electrode is shown in Fig. 11(a). Equation (11) then reduces to an equation for $V_{p l}$ which can easily be solved analytically. The solution $\delta V_{p l}(\rho)$ has cylindrical symmetry and we define $\rho$ as the radial coordinate from the center of the electrode. $\delta V_{p l}(\rho)$ is monotonically decreasing with its maximum value, $\delta V_{p l}(0)$, given by

$$
\delta V_{p l}(0)=\left(\frac{\alpha_{r}+1}{2}\right) \ln \left(\frac{\alpha_{r}+1}{2}\right) \cdot \frac{n_{s e}}{n_{0}} \cdot \frac{c_{s} e B^{2}}{2 m_{i} L_{c}} \cdot \frac{a^{2}}{\nu_{i n}} .
$$

This expression shows some interesting trends. $\delta V_{p l}(0)$ is low for a weak plasma-wall contact (low values of $n_{s e} / n_{0}$ ) or large connection length and it is inversely proportional to the ion-neutral collision frequency $\nu_{i n}$. On the other hand, $\delta V_{p l}(0)$ increases with the magnetic field strength, the surface of the electrode, and the ratio of electron to ion saturation currents.

In Fig. 11(b), the full analytical solution $\delta V_{p l}(\rho)$ is plotted for parameters relevant to our experiments (thick, blue line). Also plotted is the numerical solution (thin black line), obtained using the more realistic expressions for the parallel current given by Eqs. (1) and (2), with $V_{\text {wall }}=V_{\text {bias }}$ on the electrode and $V_{\text {wall }}=0 \mathrm{~V}$ on the grounded surfaces. Comparing the calculated values of $\delta V_{p l}$ in Fig. 11 with measurements in Fig. 6(a), we find that the estimated potential variations exceed the experimentally measured ones by a factor $\approx 10$. The parameters we assume are $n_{s e} / n_{0}=0.5$, $B=0.076 \mathrm{~T}, \quad m_{i}=1 \mathrm{amu}, \quad L_{c}=2 \pi \mathrm{m}, \quad T_{e}=2.5 \mathrm{eV}, \quad$ and $a=1.1 \mathrm{~cm}$, such that $\pi a^{2}$ corresponds approximately to the surface of two electrodes in the experiment. As in Ref. 31, we estimate $\nu_{i n}$ as $\frac{p_{n}}{T_{a m b}} \sigma^{m t} v_{t h, i}$. With a neutral pressure of $p_{n} \approx 0.02 \mathrm{~Pa}$, an ambient temperature $T_{a m b}=0.025 \mathrm{eV}$, a momentum transfer cross-section $\sigma^{m t}$ for $\mathrm{H}-\mathrm{H}^{+}$charge exchange collisions of $\approx 10^{-18} \mathrm{~m}^{2}$ and assuming an ion temperature of $1 \mathrm{eV}$ in the evaluation of the thermal velocity $v_{\text {th.i }}$, we get $\nu_{\text {in }} \approx 5 \cdot 10^{4} \mathrm{~s}^{-1}$. For the numerical solution, a bias potential $V_{\text {bias }} \gtrsim 53 \mathrm{~V}$ is necessary to assure electron saturation current over the whole surface of the electrode.

To verify if the discrepancy between experiment and our simple model can be explained by uncertainties in the assumed parameters, we look at the expression for $\delta V_{p l}(0)$ in Eq. (12). The quantity $\alpha_{r}$ is evaluated experimentally (see Fig. $7(d)$ ), justifying the assumed value $\alpha_{r} \approx 34$. The presheath density drop, on the other hand, could be reduced below the assumed value of 0.5 due to neutral friction, as discussed, e.g., in chap. 10.4 of Ref. 40. This would result in a better agreement with the experimental results. Direct measurements of $n_{s e} / n_{0}$ are planned on TORPEX to determine the significance of this effect. ${ }^{38}$ Another uncertainty is related to the ion temperature, which is not measured in the experiment. As $\delta V_{p l}(0) \propto 1 / \sqrt{T_{i}}$ and we do not expect ion temperature to exceed the assumed value of $1 \mathrm{eV}, \delta V_{p l}(0)$ should not be overestimated by this term. Finally, concentrations of impurities or molecular hydrogen ions $\left(\mathrm{H}_{2}^{+}\right.$and $\left.\mathrm{H}_{3}^{+}\right)$ are not known. This uncertainty enters as $1 /\left(m_{i} \sigma^{m t}\right)$ in Eq. (12) and would require an ion mass analyzer to be evaluated. We note here merely that the term $1 /\left(m_{i} \sigma^{m t}\right)$ does not necessarily decrease significantly with respect to the value assumed above when ions other than $\mathrm{H}^{+}$are present. As an example, we assume that collisions between $\mathrm{H}_{3}^{+}$and $\mathrm{H}_{2}$ are dominant. In this case, $m_{i}=3 \mathrm{amu}$, but at the same time, the momentum transfer cross section reduces by a factor 2-3 compared to collisions between $\mathrm{H}^{+}$and $\mathrm{H}^{48}$

While we cannot give here a definite answer on the level of uncertainty on the calculated value of $\delta V_{p l}$ in Fig. 11, the strong discrepancy of about a factor 10 compared with experimental results indicates that currents due to ion-neutral collisions should not be the main contribution to cross-field currents. This is supported by the observation that, contrary to the model, the electric field in the experiments does not everywhere point outwards of the biased flux tube, as apparent from the position of the $\delta V_{p l}$ profile (Sec. IV C).

(a)
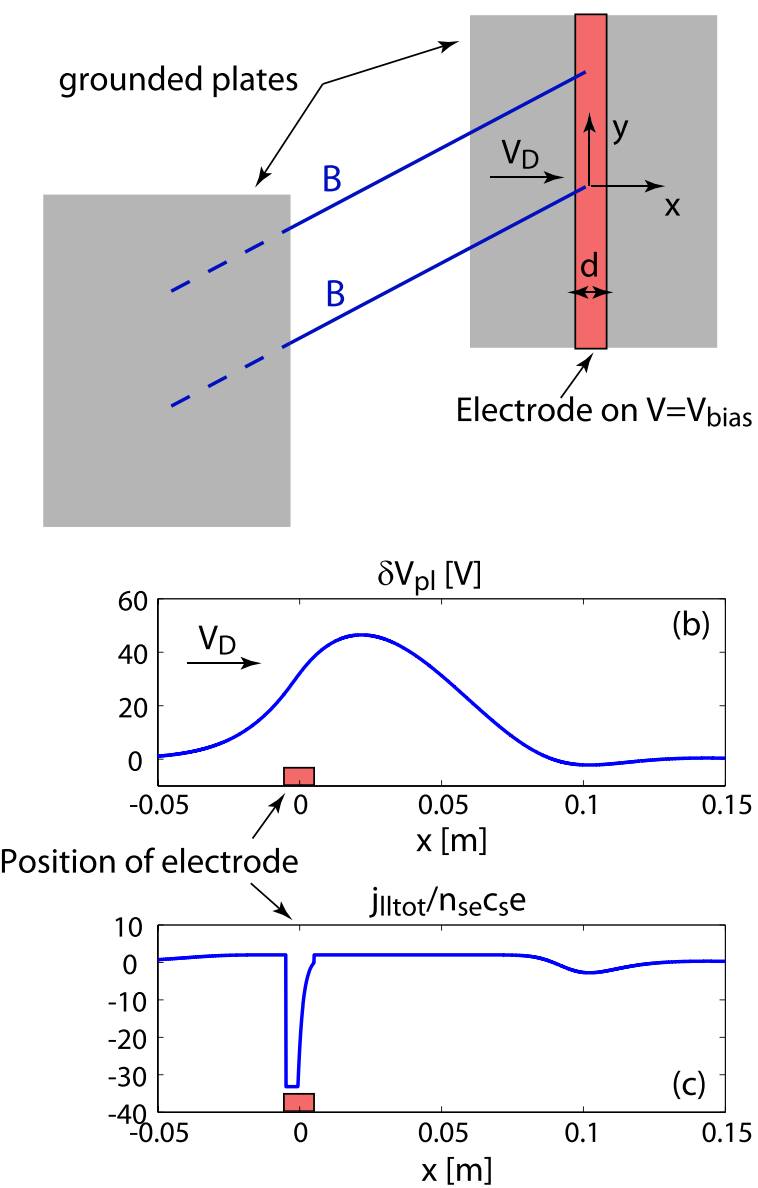

FIG. 12. Calculation of the expected plasma potential variation induced by a vertically elongated, positively biased electrode in a steady-state plasma with a uniform radial flow $V_{D}$ when ion-polarization is the only source of crossfield currents. (a) shows a sketch of the assumed geometry. (b) and (c) show the resulting plasma potential variations and total parallel current density, obtained from solving Eq. (13) for the parameters mentioned in the text. 

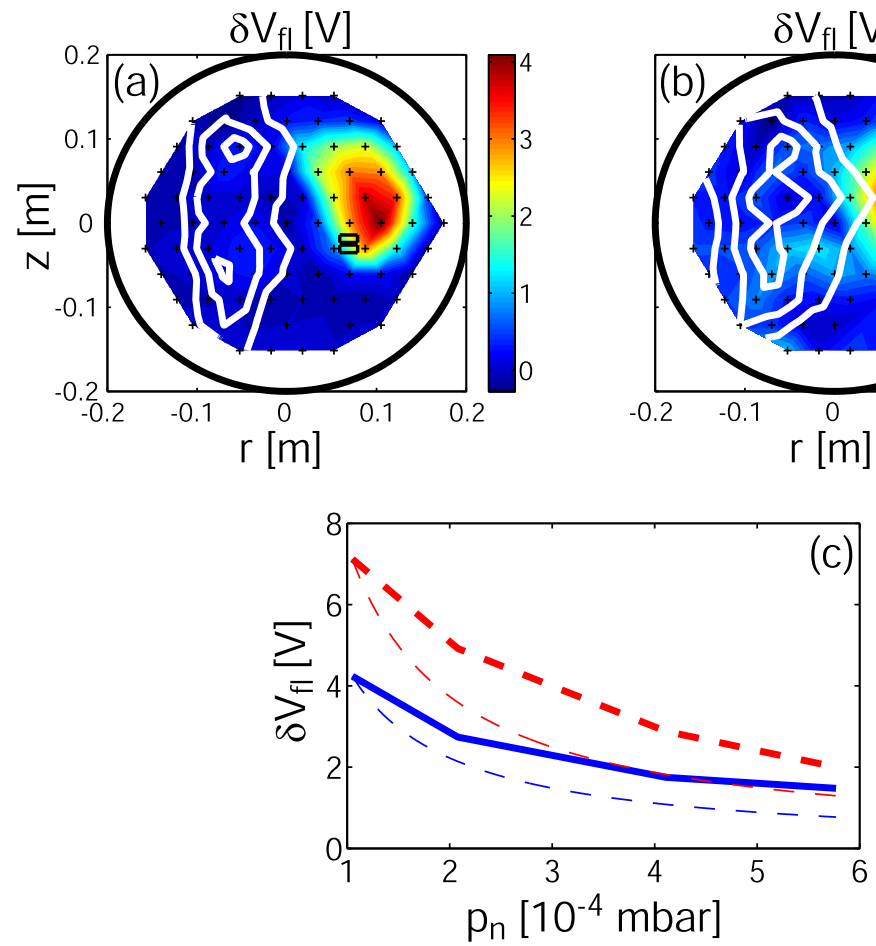

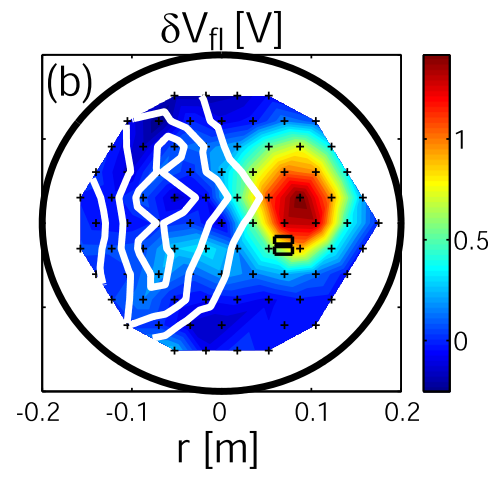

FIG. 13. $\delta V_{f l}$ for a discharge with neutral pressure $p_{n} \approx$ $10^{-4} \operatorname{mbar}(\mathrm{a})$, and $p_{n} \approx 5.8 \cdot 10^{-4} \mathrm{mbar}(\mathrm{b})$. The maximum of $\delta V_{f l}$ for four different values of $p_{n}$ is shown in (c) (thick, solid curve). The same is also plotted for a case where the bias was applied to a different pair of electrodes (thick, dashed curve). Thin, dashed curves show the $1 / p_{n}$ dependence for comparison.

\section{B. Ion polarization currents}

Next, we consider the contribution of the ionpolarization current to the cross-field current. Even in the case without fluctuations, $\partial_{t} \mathbf{E}_{\perp}=0$, which we will assume here, ion-polarization currents can be important due to the term $\left(v_{\mathbf{E} \times \mathbf{B}} \cdot \nabla_{\perp}\right) \mathbf{E}_{\perp}$. Following a similar approach to Refs. 43,46 , and 47 , we assume a vertically elongated wall electrode and a background radial cross-field ambipolar flow $v_{D}$. This is illustrated in Fig. 12(a). Experiments illustrated in Figs. 3 and 4 come closest to this. The ion-polarization term, Eq. (7), now simplifies and, assuming uniformity along the magnetic field and constant ion temperature and plasmas density, Eq. (5) becomes

$$
\partial_{x}^{3} V_{p l}=\frac{B^{2}}{n_{0} m_{i} L_{c} v_{D}} j_{\| \text {tot }}
$$

Using Eqs. (1) and (2) with $V_{\text {wall }}=V_{\text {bias }}$ on the electrode and $V_{\text {wall }}=0 \mathrm{~V}$ on the grounded surfaces, the right hand side of Eq. (13) is a function of $V_{p l}$. Equation (13) is solved numerically and in Figs. 12(b) and 12(c), the obtained radial profiles of $V_{p l}$ and $j_{\| \text {tot }}$ are plotted. We have assumed a bias potential of $+40 \mathrm{~V}$, an electrode width of $1 \mathrm{~cm}$, a background radial drift of $v_{D}=1000 \mathrm{~m} / \mathrm{s}^{-1}$, and otherwise parameters equivalent to those in Sec. V A. Again, we find absolute values of $\delta V_{p l}$ well above experimental ones. Compared to Fig. 4, we find a discrepancy of a factor 4 and this despite the fact that only half of the electrode draws an electron current, as shown in Fig. 12(c).

While this model also shows a clear discrepancy in quantitative terms compared with experiments, it shows better qualitative agreement than the previous one. Indeed, Fig. 12(b) clearly shows a shift of $\delta V_{p l}$ in the direction of the background flow.

\section{SUMMARY AND DISCUSSION}

In view of controlling SOL width and peak heat fluxes on the divertor of fusion devices by torodially (poloidally) asymmetric biasing, ${ }^{7}$ we have conducted a detailed study of biasing experiments in the TORPEX device. An array of $3 \times 8$ electrodes was installed on a conducting limiter in a region where, as in the SOL, curvature driven turbulence and blobs cause a high level of particle and heat transport across the magnetic field.

Positive biasing showed clear effects on blob propagation and time-averaged profiles. Depending on the biasing scheme, both radial and vertical blob velocities could be modified significantly. These changes were found to be quantitatively consistent with the measured changes of plasma potential.

The time-averaged perturbation of plasma potential and density profiles induced by biasing a pair of electrodes showed characteristics of a convective cell. Measurements on the limiter and over a cross-section toroidally displaced by $90^{\circ}$ were performed, showing that these perturbations are fairly uniform along the magnetic field.

Two limitations for biasing experiments have been identified. The first one is that of the locality of the induced potential variations. The strongest potential modifications are not observed along the biased flux tube, but at a position shifted in the direction of background flows. The second limitation concerns the magnitude of achievable potential variations, which are well below the potential applied to the electrodes. At the origin of this is a rather high level of cross-field currents, which is inferred from the strongly asymmetric current-voltage characteristics of the electrodes. Estimates have been performed to identify the source of these cross-field currents. Diamagnetic currents, potentially the strongest contribution, were excluded by comparison 
with the experimental profiles. The next two most promising candidates, currents due to ion-neutral friction and ionpolarization currents, were further investigated assuming simplified model equations for a steady state plasma. While the ion-polarization current case qualitatively predicts the observed shift of $\delta V_{p l}$ in the direction of plasma flows, both models give magnitudes of $\delta V_{p l}$ well above experimental values.

In contrast to these estimates, we observe a rather strong dependence of the magnitude of $\delta V_{f l}$ on the neutral pressure $p_{n}$. Fig. 13 shows results from experiments performed with four different values of neutral gas pressure, ranging from $p_{n} \approx 0.01 \mathrm{~Pa}$ to $p_{n} \approx 0.06 \mathrm{~Pa}$. The measured profiles of $\delta V_{f l}$ are shown in Figs. 13(a) and 13(b) for the two extreme cases. In (c), we show the maximum of $\delta V_{f l}$ measured with HEXTIP as a function of gas pressure for two scans, where different pairs of electrodes were used for the biasing. This shows the strong trend in $\delta V_{f l}$, which is not far from the $1 / p_{n}$ dependence indicated by the thin, dashed lines.

These observations can be compared to results presented in Refs. 49 and 50. There, experiments and simulations were performed to understand the magnitude of the negative potential well and resulting cross-field currents produced by injecting electrons into the Blaamann toroidal device using a hot negatively biased cathode. Simulations showed that the fluctuating ionpolarization current is the dominant source of cross-field current. Nevertheless, the magnitude of the potential well was found to be roughly inversely proportional to the neutral gas pressure. ${ }^{50}$

These observations point towards a complicated interplay of currents due to ion-neutral collisions and ion-polarization currents due to flows and high levels of turbulence in our experiments. Most likely, numerical simulations are required to gain more insights. Recently, two-dimensional ${ }^{45}$ and global, three-dimensional ${ }^{22}$ fluid codes have been developed for the simple magnetized torus configuration and have been validated against experiments on TORPEX..$^{51,52}$ These codes are therefore well suited to further address the question on the origin of cross-field currents.

\section{ACKNOWLEDGMENTS}

This work is supported by the Fonds National Suisse de la Recherche Scientifique. We thank M. Gilmore for helpful discussions and D. Lançon for helping with the installation of the electrodes.

${ }^{1}$ R. A. Pitts, A. Kukushkin, A. Loarte, A. Martin, M. Merola, C. E. Kessel, V. Komarov, and M. Shimada, Phys. Scr., T 138, 014001 (2009).

${ }^{2}$ A. Loarte, B. Lipschultz, A. S. Kukushkin, G. F. Matthews, P. C. Stangeby, N. Asakura, G. F. Counsell, G. Federici, A. Kallenbach, K. Krieger et al., Nucl. Fusion 47, S203 (2007).

${ }^{3}$ B. Lipschultz, X. Bonnin, G. Counsell, A. Kallenbach, A. Kukushkin, K. Krieger, A. Leonard, A. Loarte, R. Neu, R. A. Pitts et al., Nucl. Fusion 47, 1189 (2007)

${ }^{4}$ S. J. Zweben, J. A. Boedo, O. Grulke, C. Hidalgo, B. LaBombard, R. J. Maqueda, P. Scarin, and J. L. Terry, Plasma Phys. Controlled Fusion 49, S1 (2007).

${ }^{5}$ B. Labombard, J. L. Terry, J. W. Hughes, D. Brunner, J. Payne, M. L. Reinke, I. Cziegler, R. Granetz, M. Greenwald, I. H. Hutchinson et al., Phys. Plasmas 18, 056104 (2011).

${ }^{6}$ T. Eich, B. Sieglin, A. Scarabosio, W. Fundamenski, R. J. Goldston, and A. Herrmann, Phys. Rev. Lett. 107, 215001 (2011).

${ }^{7}$ R. H. Cohen and D. D. Ryutov, Nucl. Fusion 37, 621 (1997).
${ }^{8}$ I. Joseph, R. H. Cohen, and D. D. Ryutov, Phys. Plasmas 16, 052510 (2009).

${ }^{9}$ D. A. D’Ippolito, J. R. Myra, J. Jacquinot, and M. Bures, Phys. Fluids B 5, 3603 (1993).

${ }^{10}$ R. Van Nieuwenhove, G. Van Oost, P. E. Vandenplas, R. A. Moyer, D. Gray, and R. W. Conn, Fusion Eng. Des. 12, 231 (1990).

${ }^{11}$ J. Hara, Y. Uesugi, Y. Miura, and H. Kawashima, J. Nucl. Mater. 241, 338 (1997).

${ }^{12}$ G. F. Counsell, J.-W. Ahn, R. Akers, E. Arends, S. J. Fielding, P. Helander, A. Kirk, H. Meyer, A. Tabasso, H. Wilson et al., J. Nucl. Mater. 313-316, 804 (2003)

${ }^{13}$ G. F. Counsell, R. H. Cohen, P. Helander, D. D. Ryutov, and MAST Team, "Reduction of divertor power loading in MAST," in 30th EPS Conference on Controlled Fusion and Plasma Physics, St Petersburg, June 2003, Paper No. P-3.202.

${ }^{14}$ J. Stockel, P. Devynck, J. Gunn, E. Martines, G. Bonhomme, I. Voitsekhovitch, G. Van Oost, M. Hron, I. Duran, P. Stejskal et al., Plasma Phys. Controlled Fusion 47, 635 (2005).

${ }^{15}$ S. J. Zweben, R. J. Maqueda, A. L. Roquemore, C. E. Bush, R. Kaita, R. J. Marsala, Y. Raitses, R. H. Cohen, and D. D. Ryutov, Plasma Phys. Controlled Fusion 51, 105012 (2009).

${ }^{16}$ S. J. Zweben, M. D. Campanell, B. C. Lyons, R. J. Maqueda, Y. Raitses, A. L. Roquemore, F. Scotti, and H. Takahashi, "Local effects of biased electrodes in the divertor of NSTX," Plasma Phys. Controlled Fusion (submitted).

${ }^{17}$ C. Theiler, I. Furno, J. Loizu, and A. Fasoli, Phys. Rev. Lett. 108, 065005 (2012).

${ }^{18}$ A. Fasoli, B. Labit, M. McGrath, S. H. Müller, G. Plyushchev, M. Podestà, and F. M. Poli, Phys. Plasmas 13, 055902 (2006).

${ }^{19}$ A. Fasoli, A. Burckel, L. Federspiel, I. Furno, K. Gustafson, D. Iraji, B. Labit, J. Loizu, G. Plyushchev, P. Ricci et al., Plasma Phys. Controlled Fusion 52, 124020 (2010).

${ }^{20}$ M. Podestà, A. Fasoli, B. Labit, M. McGrath, S. H. Müller, and F. M. Poli, Plasma Phys. Controlled Fusion 47, 1989 (2005).

${ }^{21}$ F. M. Poli, P. Ricci, A. Fasoli, and M. Podestà, Phys. Plasmas 15, 032104 (2008).

${ }^{22}$ P. Ricci and B. N. Rogers, Phys. Rev. Lett. 104, 145001 (2010).

${ }^{23}$ D. A. D'Ippolito, J. R. Myra, and S. J. Zweben, Phys. Plasmas 18, 060501 (2011).

${ }^{24}$ S. I. Krasheninnikov, D. A. D'Ippolito, and J. R. Myra, J. Plasma Phys. 74, 679 (2008).

${ }^{25}$ O. E. Garcia, Plasma Fusion Res. 4, 19 (2009).

${ }^{26}$ I. Furno, B. Labit, M. Podestà, A. Fasoli, S. H. Müller, F. M. Poli, P. Ricci, C. Theiler, S. Brunner, A. Diallo et al., Phys. Rev. Lett. 100, 055004 (2008).

${ }^{27}$ S. H. Müller, A. Diallo, A. Fasoli, I. Furno, B. Labit, and M. Podestà, Phys. Plasmas 14, 110704 (2007).

${ }^{28}$ C. Theiler, A. Diallo, A. Fasoli, I. Furno, B. Labit, M. Podestà, F. M. Poli, and P. Ricci, Phys. Plasmas 15, 042303 (2008).

${ }^{29}$ I. Furno, B. Labit, A. Fasoli, F. M. Poli, P. Ricci, C. Theiler, S. Brunner, A. Diallo, J. P. Graves, M. Podestà et al., Phys. Plasmas 15, 055903 (2008).

${ }^{30}$ C. Theiler, I. Furno, P. Ricci, A. Fasoli, B. Labit, S. H. Müller, and G. Plyushchev, Phys. Rev. Lett. 103, 065001 (2009).

${ }^{31}$ C. Theiler, I. Furno, A. Fasoli, P. Ricci, B. Labit, and D. Iraji, Phys. Plasmas 18, 055901 (2011).

${ }^{32}$ I. Furno, M. Spolaore, C. Theiler, N. Vianello, R. Cavazzana, and A. Fasoli, Phys. Rev. Lett. 106, 245001 (2011).

${ }^{33}$ M. Podestà, A. Fasoli, B. Labit, I. Furno, P. Ricci, F. M. Poli, A. Diallo, S. H. Müller, and C. Theiler, Phys. Rev. Lett. 101, 045001 (2008).

${ }^{34}$ B. Labit, C. Theiler, A. Fasoli, I. Furno, and P. Ricci, Phys. Plasmas 18, 032308 (2011).

${ }^{35}$ S. H. Müller, A. Fasoli, B. Labit, M. McGrath, O. Pisaturo, G. Plyushchev, M. Podestà, and F. M. Poli, Phys. Plasmas 12, 090906 (2005).

${ }^{36}$ H. Johnsen, H. L. Pécseli, and J. Trulsen, Phys. Fluids 30, 2239 (1987).

${ }^{37}$ G. R. Tynan, L. Schmitz, R. W. Conn, R. Doerner, and R. Lehmer, Phys. Rev. Lett. 68, 3032 (1992).

${ }^{38} \mathrm{C}$. Theiler, "Basic investigation of turbulent structures and blobs of relevance for magnetic fusion plasmas," Ph.D. dissertation, No. 5228 (Ecole Polytechnique Federale de Lausanne (EPFL), 2011).

${ }^{39}$ C. Theiler, I. Furno, A. Kuenlin, P. Marmillod, and A. Fasoli, Rev. Sci. Instrum. 82, 013504 (2011).

${ }^{40}$ P. C. Stangeby, The Plasma Boundary of Magnetic Fusion Devices (Institute of Physics, Berkshire, 2000).

${ }^{41}$ D. D. Ryutov, P. Helander, and R. H. Cohen, Plasma Phys. Controlled Fusion 43, 1399 (2001). 
${ }^{42}$ R. H. Cohen, S. Fielding, P. Helander, and D. D. Ryutov, Contrib. Plasma Phys. 42, 296 (2002).

${ }^{43}$ V. A. Rozhansky, in Reviews of Plasma Physics, edited by V. D. Shafranov (Springer-Verlag, Berlin/Heidelberg, 2008), Chap. 1, Vol. 24.

${ }^{44}$ A. Zeiler, J. F. Drake, and B. Rogers, Phys. Plasmas 4, 2134 (1997).

${ }^{45}$ P. Ricci, B. N. Rogers, and S. Brunner, Phys. Rev. Lett. 100, 225002 (2008).

${ }^{46}$ V. A. Rozhansky, A. A. Ushakov, and S. P. Voskoboynikov, Nucl. Fusion 39, 613 (1999).

${ }^{47}$ A. Carlson, Phys. Plasmas 8, 4732 (2001).
${ }^{48}$ Atomic and Molecular Processes in Fusion Edge Plasmas, edited by R. K. Janev (Plenum, New York, 1995).

${ }^{49}$ K. Rypdal, O. E. Garcia, and J.-V. Paulsen, Phys. Rev. Lett. 79, 1857 (1997).

${ }^{50}$ K. Rypdal, J. V. Paulsen, and O. E. Garcia, Czech. J. Phys. 48, 207 (1998). ${ }^{51}$ P. Ricci, C. Theiler, A. Fasoli, I. Furno, B. Labit, S. H. Müller, M. Podestà, and F. M. Poli, Phys. Plasmas 16, 055703 (2009).

${ }^{52}$ P. Ricci, C. Theiler, A. Fasoli, I. Furno, K. Gustafson, D. Iraji, and J. Loizu, Phys. Plasmas 18, 032109 (2011). 